\title{
Novel Aspects in Manufacture, Storage, and Transfusion of Blood Components
}

\author{
Peter Schlenke \\ Department of Blood Group Serology and Transfusion Medicine, Medical University of Graz, Graz, Austria
}

I am very pleased to inform you that the very recently published impact factor of the journal Transfusion Medicine and Hemotherapy is 3.000. First of all, I would like to congratulate everybody involved in this success, particularly the distinguished authors and scientists around the world willing to publish in the journal during the transition phase into a well-recognized international journal. This was only possible thanks to the extraordinary commitment of a handful of associate editors and their professional expertise and personal enthusiasm. Karger Publishers has made a valuable and sustainable contribution supporting this evolutionary development into a high-quality journal.

Nowadays, the scientific community is becoming aware that journal impact factors have some noteworthy limitations, particularly for the evaluation of individual academic careers. Nonetheless, Transfusion Medicine and Hemotherapy is gaining more visibility and reputation in the field of "blood issues." The journal is now more attractive for an international readership as well as for submissions of articles in a worldwide competitive environment of peer-reviewed publication organs.

However, there are more scientists, more knowledge production and even more papers than ever on the market, which call for innovative strategies and ethical principles to ensure the compliance with the standards of Good Scientific Practice and with a quality-assured peerreview process by international experts in the field negating any major conflicts of interest.

The journal impact factor is a bibliometric index reflecting the number of citations (in a 2-year period) in relation to the number of "citable" articles published in the same time frame and is considered as an important measure for scientific quality.

Today, other bibliometric measures, such as the 5-year impact factor, the impact factor without self-citations and the citing half time are in use in order to improve the scientific significance. In this context, it is important to stress that the journal impact factor is per se vulnerable either to statistical outliers (only very few articles with a high citation rate contribute to the rise of the impact factor) or to "targeted" interventions such as a high proportion of self-citations or journal citation requests.

From a different point of view, the scientific community should realize that in general the citation rate - in contrast to Science or Nature - of articles published in journals comparable in the ranking position with Transfusion Medicine and Hemotherapy is astonishingly low, which might reflect an imbalance in the acceptable amount of citations per article (e.g., 20-40) and an inflationary trend of annually published "items" rather than the release of scientifically insignificant publications per se. This is especially true for publications on subsidiary subjects or in highly specialized fields with a smaller community of scientists globally involved.

Based on the general debate on the validity of the impact factor, Transfusion Medicine and Hemotherapy will undertake substantial efforts to target a selection of highquality manuscripts in the field of transfusion medicine and neighboring disciplines such as hemostaseology and cell therapy for publication, which will improve the quality and relevance of the journal in the most genuine manner. I would like to encourage all readers and authors to keep an eye on Transfusion Medicine and Hemotherapy.

The "hot topic" of the current issue is entitled "Novel Aspects in Manufacture, Storage and Transfusion of Blood Components" containing a basket of research articles and a meta-analysis which all became accepted within the last couple of months and will now be published together as print edition composed by a very good chance of a broader theme. Convenient to the occasion of the new journal

\section{KARGER}

(C) 2019 S. Karger AG, Basel 
impact factor, Transfusion Medicine and Hemotherapy is proud to offer their international readership a total of six scientific contributions written by authors employed around the world (Europe, Iran, India, China, USA).

Blood is a lifesaving liquid organ collected from more than 100 million individuals per year, mainly from voluntary nonremunerated blood donors in order to cover the blood demand of a global population of about 7 billion people as estimated in the Global Status Report on Blood Safety and Availability 2016 by the World Health Organization [1]. In high-income countries, the therapy with specific blood components is most commonly used for patients undergoing major surgery such as cardiovascular, transplant or visceral surgery, for polytrauma patients with massive hemorrhage or for patients suffering from solid cancer and hematological malignancies. In contrast, in developing countries, the most important indication to give blood is severe childhood anemia due to malaria. As treatment with blood can transmit harmful infectious diseases such as hepatitis, HIV/AIDS, and bacterial sepsis, the entire manufacturing process ("from vein to vein") should be properly designed considering international standards (e.g., Good Manufacturing Practice) and supervised by both an internally established quality management system and by national authorities. In today's edition, the paper written by Dimberg et al. [2] dealt with the pathogen inactivation of whole blood donations (Mirasol system) using riboflavin (vitamin $\mathrm{B}_{2}$ ) and ultraviolet light with a higher energy dose of $80 \mathrm{~J} / \mathrm{mL}$ and its subsequent influence on red blood cell (RBC) quality during a limited storage time of 21 days. Valkonen et al. [3] manufactured buffy coat-derived pooled platelet concentrates by using different platelet additive solutions (PAS B and E [supplemented with phosphate, potassium and magnesium]) and investigated the level of platelet activation by a variety of parameters including CD62P exposure, soluble glycoprotein $\mathrm{V}$ content and the concentration of CD41 expressing extracellular vesicles using a sophisticated nanoparticlebased tracking technology. The research of Hsieh et al. [4] focused on the well-known changes in physical and biochemical properties during the aging of RBCs which are closely linked to the so-called storage lesion. They dealt in their contribution with the particular question to what extent physiologically younger RBCs are more robust with respect to the above-mentioned alterations and have a higher tolerance against oxidative stress when compared to old RBCs. In their current study, Hashemi Tayer et al. [5] investigated the formation of RBC-derived extracellular microvesicles upon a shelf life of 42 days and demonstrated a nearly 20 -fold increase in gylcophorin A and annexin V-positive microvesicles during a 6-week period. Furthermore, the procoagulant activity of externalized phosphatidylserine was appropriately measured by an ELISA assay and abolished by microvesicle filtration. This age-related hypercoagulable state of stored RBCs is per se an interesting observation and it needs to be clarified how it might contribute to thromboembolic complications especially in patient cohorts at risk. However, the clinical impact of the RBC storage lesion on morbidity and mortality is still controversial. Zhang et al. [6] published in this issue of Transfusion Medicine and Hemotherapy a metaanalysis including six randomized clinical trials that enrolled a total of 8,467 critically ill patients and calculated the risk ratio with $95 \%$ confidence intervals. There were no significant differences in the primary endpoint (ICU mortality) and further secondary endpoints (e.g., in-hospital mortality) with "fresh" versus "old" RBC transfusions. Besides the manufacturing of cellular blood components, plasma derivates such as albumins, coagulation factors and immunoglobulins could be generated in plasma fractionation centers of licensed pharmaceutical companies. De Angelis and Breda [7] compared in their health economic article the domestic plasma fractionation in Italy after market liberalization. They concluded that effective competition leads to a higher degree of flexibility (portfolio of medicines), higher yields of albumin and immunoglobulins and lower price levels and facilitates the national strategy for self-sufficiency in plasma-derived medicinal products.

We would like to invite you to share this published knowledge on novel aspects in manufacture, storage and transfusion of blood components.

\section{References}

1 World Health Organization. Global status report on blood safety and availability 2016 . Geneva: World Health Organization; 2017.

2 Dimberg LY, Doane SK, Yonemura S, Reddy HL, Hovenga N, Gosney E.J, Tran M, Wilkinson S, Goodrich RP, Marschner S. Red Blood Cells Derived from Whole Blood Treated with Riboflavin and UV Light Maintain Adequate Cell Quality through 21 Days of Storage. Transfus Med Hemother. 2019;46(4). DOI: https://doi.org/10.1159/000495257.

3 Valkonen S, Mallas B, Impola U, Valkeajärvi A, Eronen J, Javela K, Siljander PR-M, Laitinen S. Assessment of Time-Dependent Platelet
Activation Using Extracellular Vesicles, CD62P Exposure, and Soluble Glycoprotein V Content of Platelet Concentrates with Two Different Platelet Additive Solutions. Transfus Med Hemother. 2019;46(4). DOI: https:// doi.org/10.1159/000499958.

4 Hsieh C, Prabhu NCS, Rajashekaraiah V. Age-Related Modulations in Erythrocytes under Blood Bank Conditions. Transfus Med Hemother. 2019;46(4). DOI: https://doi. org/10.1159/000501285.

5 Hashemi Tayer A, Amirizadeh N, Ahmadinejad M, Nikougoftar M, Deyhim MR, Zolfaghari S. Procoagulant Activity of Red
Blood Cell-Derived Microvesicles during Red Cell Storage. Transfus Med Hemother. 2019;46(4). DOI: https://doi.org/10.1159/ 000494367.

6 Zhang W, Yu K, Chen N, Chen M. Age of Red Cells for Transfusion and Outcomes in Critically Ill Patients: A Meta-Analysis. Transfus Med Hemother. 2019;46(4). DOI: https://doi. org/10.1159/000498863.

7 De Angelis V, Breda A. Trends in Plasma Toll Fractionation for Self Sufficiency of PlasmaDerived Medicinal Products in Italy. Transfus Med Hemother. 2019;46(4). DOI: https://doi. org/10.1159/000496750. 